\title{
Geomagnetically Induced Currents as Ground Effects of Space Weather
}

\author{
Risto Pirjola \\ ${ }^{1}$ Finnish Meteorological Institute \\ ${ }^{2}$ Natural Resources Canada \\ ${ }^{1}$ Finland \\ ${ }^{2}$ Canada
}

\section{Introduction}

"Space Weather" refers to electromagnetic and particle conditions in the near-Earth space. It is controlled by solar activity. The whole space weather chain extending from the Sun to the Earth's surface is very complicated and includes plasma physical processes, in which the interaction of the solar wind with the geomagnetic field plays an essential role. Space weather phenomena statistically follow the eleven-year sunspot cycle but large space weather storms can also occur during sunspot minima. Changes of currents in the Earth's magnetosphere and ionosphere during a space weather storm produce temporal variations of the geomagnetic field, i.e. geomagnetic disturbances and storms. Technological systems, even humans, in space and on the ground may experience adverse effects from space weather (e.g. Lanzerotti et al., 1999).

At the Earth's surface, space weather manifests itself as "Geomagnetically Induced Currents" (GIC) in technological conductor networks, such as electric power transmission grids, oil and gas pipelines, telecommunication cables and railway circuits. GIC observations have a much longer history than the time when the concept of space weather has been used as GIC effects were already found in the first telegraph equipment in the mid-1800's (Boteler et al., 1998; Lanzerotti et al., 1999; Lanzerotti, 2010). Telecommunication systems have suffered from GIC problems several times in the past. Optical fibre cables generally used today are not directly affected by space weather. However, metal wires lying in parallel with fibre cables are used to provide power to repeater stations, and they may be prone to GIC impacts. Trans-oceanic submarine communication cables are a special category regarding GIC since their lengths imply that the end-to-end voltages associated with GIC can be very large.

Buried pipelines may suffer from serious corrosion of the steel due to GIC (e.g. Gummow, 2002). Corrosion is an electrochemical process occurring at points where a current flows from the pipe to the soil. Roughly speaking, a continuous dc current of $1 \mathrm{~A}$ for one year causes a loss of about $10 \mathrm{~kg}$ of steel. To prevent or minimise corrosion, so-called cathodic protection (CP) systems are used for pipelines. They keep the pipeline in a negative voltage of typically slightly less than $1 \mathrm{~V}$ with respect to the soil. Pipe-to-soil voltages associated with GIC may well exceed the $\mathrm{CP}$ voltage, thus possibly cancelling and invalidating the protection. Furthermore, control surveys of pipe-to-soil voltages during space weather storms may lead to 
incorrect data. Pipelines are covered with a highly-resistive coating, whose materials have a much larger resistivity today than in earlier times. But a high resistance also increases pipe-tosoil voltages implying larger harmful currents at possible defects in the coating.

GIC impacts on railways have not been much investigated yet but evidence of anomalies in railway signalling systems due to GIC exists at least in Sweden and Russia (Ptitsyna et al., 2008; Wik et al., 2009; Eroshenko et al., 2010).

Nowadays electric power transmission networks are the most important regarding GIC effects, and the importance continuously increases with the extension of power grids including complex continent-wide interconnections and with the even larger dependence of the society on the availability and reliability of electricity. The frequencies associated with GIC are typically very much lower than the $50 / 60 \mathrm{~Hz}$ ac frequency used in power transmission. Thus, from the viewpoint of a power system, GIC are (quasi-)dc currents. Consequently their presence may lead to half-cycle saturation of transformers, which can result in non-linear behaviour of transformers (e.g. Molinski, 2002; Kappenman, 2007). This further implies large asymmetric exciting currents producing harmonics, unnecessary relay trippings, increased reactive power demands, voltage fluctuations, and possibly even a collapse of the whole power network. Transformers can also be overheated with possible damage. The best-known GIC disturbance is a province-wide blackout in Québec, Canada, for several hours during a large geomagnetic storm in March 1989 (e.g. Bolduc, 2002). A transformer was permanently damaged and had to be replaced in New Jersey, USA, during the same storm (Kappenman \& Albertson, 1990).

All this means that research of GIC and space weather is not only relevant and significant regarding space science but important practical applications also exist. As indicated above, today's GIC research is particularly concentrated on power networks, which constitute the main focus in this paper as well. The discussion is limited to space physical and geophysical aspects associated with GIC including the calculation of GIC but neglecting the consideration of engineering details of possible adverse impacts of GIC on networks and their equipment and the discussion of mitigation means against GIC problems.

The shape of the geomagnetic field implies that geomagnetic storms are the most intense and most frequent at high latitudes. So GIC are a special concern in the same areas. However, during major space weather storms, large geomagnetic disturbances may also occur at much lower latitudes, which indicates the possibility of GIC problems there, too. Moreover, GIC magnitudes in a system depend significantly on the network topology, configuration and resistances. GIC values also vary much from site to site in a system being generally large at ends and corners of a network. In addition, the sensitivity of a system to GIC depends on many technical matters and varies from one network to another. Consequently, a GIC value that can be ignored in one system may be hazardous in another. All this shows that GIC issues have to be taken into account in mid- and low-latitude networks, too (e.g. Kappenman, 2003; Trivedi et al., 2007; Bernhardi et al., 2008; Liu et al., 2009a, 2009b).

Finland is located at high latitudes. Consequently, GIC would be a potential problem in the country, and in fact, research of GIC has been carried out as collaboration between Finnish power and pipeline industry and the Finnish Meteorological Institute since the latter part of the 1970's. However, fortunately, GIC have never caused significant problems in Finland 
(Elovaara et al., 1992; Elovaara, 2007) whereas the neighbour country, Sweden, has experienced harmful GIC effects several times (Elovaara et al., 1992; Pirjola \& Boteler, 2006; Wik et al., 2009). Such a dissimilarity between Sweden and Finland is somewhat surprising but, concerning power systems, it can be explained based on differences in transformer design and specifications in the two countries.

In Section 2 of this paper, we consider research of GIC, which can be done by measurements or by theoretical modelling. Section 3 is devoted to a more detailed discussion of the calculation of GIC in power networks and pipelines, and Section 4 contains concluding remarks.

\section{Research of GIC}

Research of GIC is highly multidisciplinary since the subjects involved cover items from solar physics to engineering details of the operation of power systems or other networks. We often speak about the "space weather chain" that begins at solar activity, extends via the solar wind and magnetospheric-ionospheric processes to GIC in ground-based systems and to the mitigation of adverse effects of GIC (e.g. Pirjola, 2000; Pirjola et al., 2003). Roughly speaking, GIC studies can be divided into two parts, the first of which refers to the space physical and geophysical investigation of GIC in a network, whereas the second part includes the engineering evaluation of effects of GIC on the system in question as well as the design of techniques for mitigating the harmful impacts. This paper deals with the first part.

The flow of GIC in a network is easy to understand based on Faraday's and Ohm's laws. The geomagnetic field experiences temporal variations during a space weather event. According to Faraday's law, they are accompanied by a geoelectric field, which, based on Ohm's law, drives currents in conductors, i.e. GIC in networks. In theoretical discussions, the determination of GIC in a system is usually divided into two parts or steps. The "geophysical part" and the "engineering part" refer to the modelling of the horizontal geoelectric field at the Earth's surface and to the calculation of GIC in the particular network, respectively (e.g. Pirjola, 2002).

GIC can naturally be studied by making measurements or by theoretical modelling. In practice, the validity of the models always has to be verified by measured data. If necessary, the data may enable adjusting model parameter values. Concerning an appropriate model of the Earth's conductivity in southern Sweden, the adjustment is explicitly demonstrated by Wik et al. (2008).

\subsection{Measurements of GIC}

The usual place for installing GIC recording equipment in a power network is the earthing lead of a transformer neutral. In the normal situation, this particular lead carries no 50/60 $\mathrm{Hz}$ current because the sum of the ac currents in the three phases is equal to zero. Furthermore, measuring GIC in the neutral lead directly gives information about the currents flowing through transformer windings, where they can result in harmful saturation. Recordings of GIC can be performed with a coil around the earthing lead. However, for example in the Finnish $400 \mathrm{kV}$ network, a small shunt resistor is utilised in the lead (e.g. Elovaara et al., 1992). The largest GIC magnitudes measured in Finland and in 

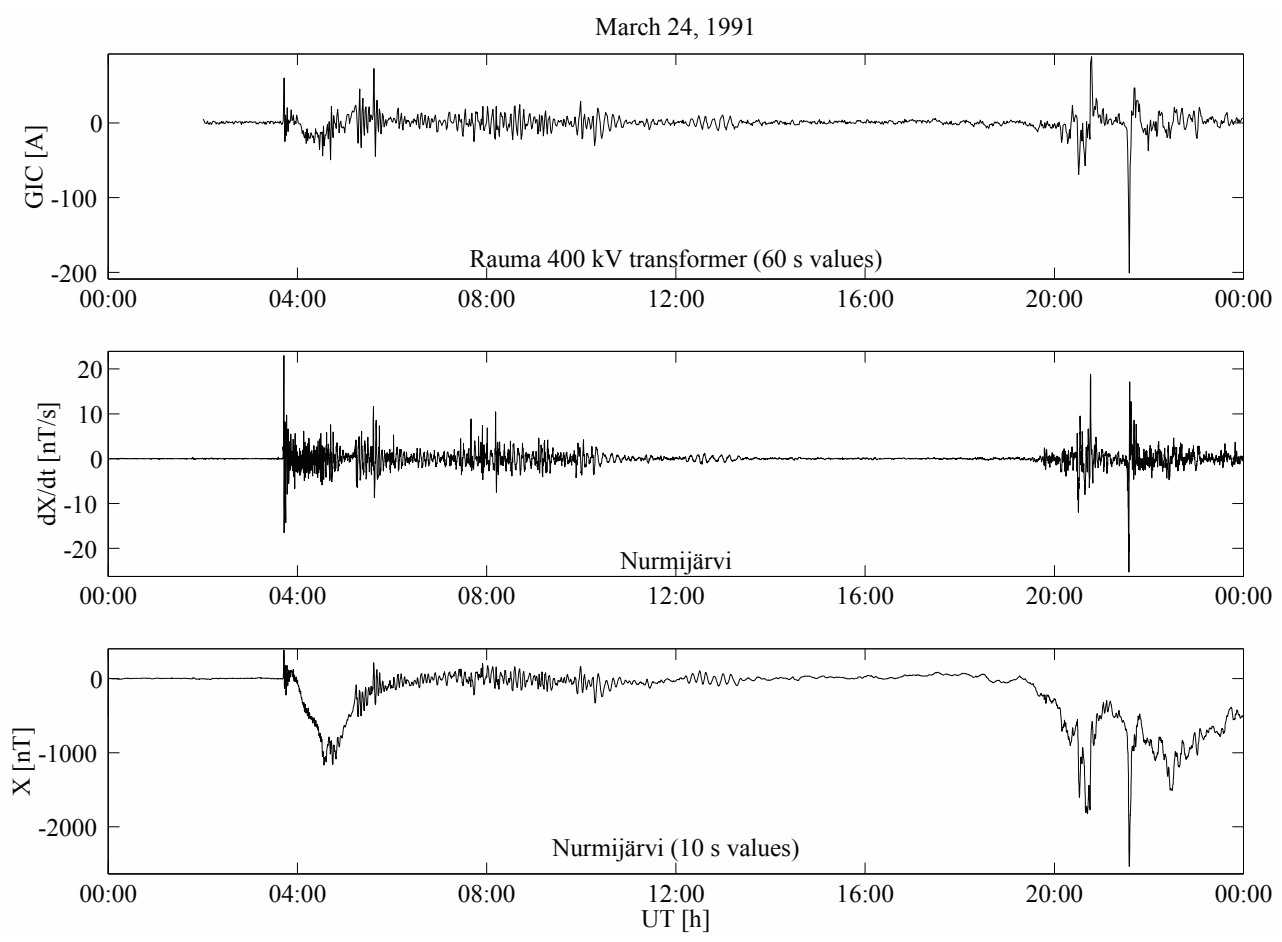

Fig. 1. (Top) GIC recorded in the earthing lead of the Rauma $400 \mathrm{kV}$ transformer neutral in southwestern Finland on March 24, 1991, (Bottom) north component (X) of the geomagnetic field, and (Middle) its time derivative at the Nurmijärvi Geophysical Observatory in southern Finland. The value of 201 A seen in the top plot is the largest measured in the GIC recordings in the Finnish 400 kV system started in 1977 (Pirjola et al., 2003; Pirjola et al., 2005).

Sweden are about $200 \mathrm{~A}$ and about 300 A, respectively (Pirjola et al., 2005; Wik et al., 2008). It should be noted that these values give the total GIC, i.e. the sum of GIC in the three phases. The per-phase values are obtained by dividing by three, being thus about 67 $\mathrm{A}$ and $100 \mathrm{~A}$. To our knowledge, the Swedish value is the highest ever reported. It occurred during a great geomagnetic storm in April 2000. Figure 1 presents GIC recorded in the earthing lead of the Rauma $400 \mathrm{kV}$ transformer neutral in southwestern Finland on March 24, 1991. It also includes the above-mentioned largest value of about $200 \mathrm{~A}$ just before $22 \mathrm{~h}$ Universal Time (UT).

The bottom and middle plots of Figure 1 show the recordings of the north component (denoted by $\mathrm{X}$ ) of the geomagnetic field and its time derivative at the Nurmijärvi Geophysical Observatory located in southern Finland at a distance of about $200 \mathrm{~km}$ from Rauma. We see that the GIC curve resembles the derivative, but the behaviour of the geomagnetic field is more different. This would seem to be an expected result because GIC are created based on Faraday's law, in which temporal variations of the magnetic field play 
a role. However, it is important to note that the geoelectric field and GIC are not directly proportional to the geomagnetic time derivative but the relation is more complicated (see Section 2.2). Pirjola (2010) investigates the relation between geomagnetic variations and the geoelectric field (and GIC) in the case of a simple two-layer Earth. He explicitly shows that a poorly-conducting upper layer above a highly-conducting bottom is favourable to the geoelectric field (and GIC) being proportional to the geomagnetic time derivative at the Earth's surface whereas a thin highly-conducting upper layer above a less-conducting bottom results in the surface geoelectric field (and GIC) proportional to the geomagnetic variation. Trichtchenko \& Boteler $(2006 ; 2007)$ present an example from Canada in which GIC resembles the geomagnetic variation and another example also from Canada in which a correspondence between GIC and the geomagnetic time derivative exists. Watari et al. (2009) demonstrate that in recordings in the Japanese power network GIC show a high correlation with the geomagnetic variation field rather than with the geomagnetic time derivative.

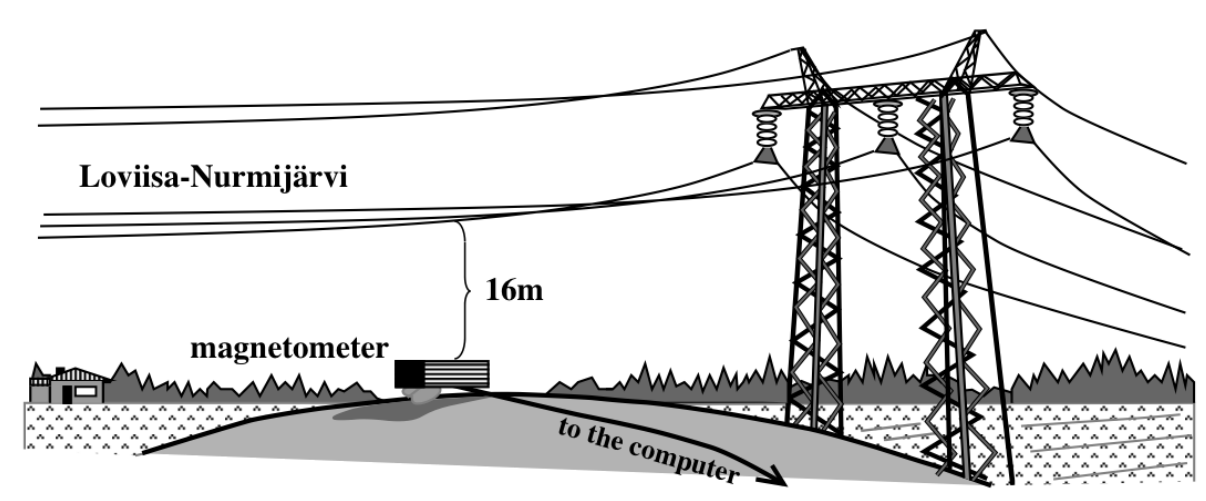

Fig. 2. Measurement of GIC flowing in a power transmission line by using a magnetometer below the line and another magnetometer for reference data further away (Mäkinen, 1993; Viljanen et al., 2009).

Although GIC in transformer neutral earthing leads have a larger practical significance than GIC in transmission lines, both are of the same importance from the scientific point of view. This is a motivation for the measurements of GIC carried out in a transmission line in Finland for some time in the beginning of the 1990's (Mäkinen, 1993; Viljanen et al., 2009). Such measurements are possible to make by using two magnetometers, which behave slowly enough, so that the influence from the ac currents is not experienced. One magnetometer is installed very near the line, as shown in Figure 2. The other is located further away. The former records the sum of the natural geomagnetic field and the field due to GIC in the line whereas the latter only observes the natural field. If the distance of the two magnetometers is not too large (i.e. many tens of kilometres or more), the natural field can be regarded as the same at the two sites. Thus the difference of the magnetometer readings gives the field created by GIC flowing in the line, and by inverting the Biot-Savart law GIC data are obtained. It is naturally necessary to take into account that a transmission line consists of three phase conductors, each of which carries one third of the (total) GIC. The 
geometries of the phases with respect to the nearby magnetometer are different, which must also be taken into account in the inversion.

A similar two-magnetometer technique has been used successfully to record GIC flowing along the Finnish natural gas pipeline since 1998 (Pulkkinen et al., 2001a; Viljanen et al., 2006). The largest GIC recorded so far is 57 A on October 29, 2003 (Pirjola et al., 2005). The pipe-to-soil voltage is also continuously monitored in the Finnish pipeline as well as in other oil and gas pipelines.

\subsection{Modelling of GIC}

As pointed out in the beginning of Section 2, GIC modelling is convenient to be carried out in two parts:

1. Determination of the horizontal geoelectric field at the Earth's surface ("geophysical part").

2. Computation of GIC in the network produced by the geoelectric field ("engineering part").

The geophysical part does not depend on the particular network and is thus the same for power networks, pipelines and other conductor systems. The input of the geophysical part consists of knowledge or assumptions about the Earth's conductivity and about the magnetospheric-ionospheric currents or about the geomagnetic variations at the Earth's surface. The solution is based on Maxwell's equations

$$
\begin{gathered}
\nabla \cdot \mathbf{E}=\frac{\rho}{\varepsilon_{0}} \\
\nabla \cdot \mathbf{B}=0 \\
\nabla \times \mathbf{E}=-\frac{\partial \mathbf{B}}{\partial t} \\
\nabla \times \mathbf{B}=\mu_{0} \mathbf{j}+\mu_{0} \varepsilon_{0} \frac{\partial \mathbf{E}}{\partial t}
\end{gathered}
$$

Maxwell's equations couple the electric field $\mathbf{E}[\mathrm{V} / \mathrm{m}]$ and the magnetic field $\mathbf{B}\left[\mathrm{Vs} / \mathrm{m}^{2}\right]$ to each other as well as to the charge density $\rho\left[\mathrm{As} / \mathrm{m}^{3}\right]$ and to the current density $\mathbf{j}\left[\mathrm{A} / \mathrm{m}^{2}\right]$. All these quantities are functions of space $\mathbf{r}$ and time $t$. The vacuum permeability and the vacuum permittivity are denoted by $\mu_{0}\left(=4 \pi \cdot 10^{-7} \mathrm{Vs} / \mathrm{Am}\right)$ and $\varepsilon_{0}\left(=8.854 \cdot 10^{-12} \mathrm{As} / \mathrm{Vm}\right)$, respectively. In the form (1)-(4), Maxwell's equations are (microscopically) always valid. However, charges and currents are macroscopically usually divided into different types, and additional fields are introduced. The electric $\mathbf{D}$ field is related to $\mathbf{E}$ and the magnetic $\mathbf{H}$ field is related to $\mathbf{B}$ by constitutive equations, which are usually assumed to be linear and simple as follows

$$
\begin{aligned}
& \mathbf{D}=\varepsilon \mathbf{E} \\
& \mathbf{H}=\frac{\mathbf{B}}{\mu}
\end{aligned}
$$


where $\mu$ and $\varepsilon$ are the permeability and permittivity of the medium. The usual assumption in connection with geoelectromagnetic studies is that $\mu=\mu_{0}$. Macroscopically, we can write Maxwell's equations similarly to (1)-(4) but $\mu_{0}$ and $\varepsilon_{0}$ are replaced by $\mu$ and $\varepsilon_{\text {, }}$ and the charge density $\rho=\rho_{\text {free }}$ and the current density $\mathbf{j}=\mathbf{j}_{\text {free }}$ should only refer to charges moving freely in the medium. An additional constitutive equation needed in the geophysical part is the Ohm's law that relates $\mathbf{j}_{\text {free }}$ and $\mathbf{E}$

$$
\mathbf{j}_{\text {free }}=\sigma \mathbf{E}
$$

where $\sigma$ is the conductivity of the medium. What is still required for solving the geophysical part are the continuity conditions that enable moving from one medium to another. Usually we utilise the continuity of the tangetial components of the $\mathbf{E}$ and $\mathbf{H}$ fields when moving across a boundary.

Different techniques and models for performing the geophysical part have been investigated for a long time (e.g. Pirjola, 2002, and references therein). An interesting approximate alternative is the Complex Image Method (CIM), in which the currents induced in the conducting Earth are replaced by images of ionospheric currents located in a complex space (Boteler \& Pirjola, 1998a; Pirjola and Viljanen, 1998). A crucial parameter in CIM is the complex skin depth $p=p(\omega)$, which depends on the angular frequency $\omega$ considered (i.e. we assume a harmonic dependence on the time $t$ given by $\exp (\mathrm{i} \omega t))$

$$
p(\omega)=\frac{Z(\omega)}{i \omega \mu_{0}}
$$

where $Z=Z(\omega)$ is the surface impedance at the Earth's surface relating a horizontal electric field component $E_{y}=E_{y}(\omega)$ to the perpendicular horizontal magnetic field component $B_{x}=B_{x}(\omega)$ (see e.g. Kaufman \& Keller, 1981; Pirjola et al., 2009)

$$
E_{y}(\omega)=-\frac{Z(\omega)}{\mu_{0}} B_{x}(\omega)
$$

It is implicitly required in equation (9) that the (flat) Earth surface is the $x y$ plane of a righthanded Cartesian coordinate system in which the $z$ axis points downwards. In practice, the surface impedance included in equation (9) and especially in equation (8) refers to the plane wave case discussed briefly below.

CIM makes numerical computations much faster than with formulas obtained by a direct forward solution of Maxwell's equations and boundary conditions (Häkkinen \& Pirjola, 1986; Pirjola \& Häkkinen, 1991). However, it has been proved that, regarding the accuracy and fastness of computations required in GIC applications, the simple plane wave method to be applied locally in different areas covered by the particular network is the best and most practical technique (Viljanen et al., 2004). It should also be noted that, in GIC calculations, it is not necessary to know the spatial details of the geoelectric field exactly because the (geo)voltages driving GIC are obtained by integrating the geoelectric field (see Section 3.1).

In the plane wave method, the geoelectromagnetic disturbance produced by the (primary) magnetospheric-ionospheric currents is a plane wave propagating vertically downwards and the Earth's conductivity structure is layered (with a flat surface) enabling the Earth to be 
described by a surface impedance (see equation (9)). The contribution to the total geoelectromagnetic disturbance at the Earth's surface from (secondary) currents in the Earth is an upward-propagating reflected wave. This kind of a model is already included in the basic paper of magnetotellurics by Cagniard (1953). It is necessary to emphasise that the frequencies involved in geoelectromagnetic studies are typically in the $\mathrm{mHz}$ range and at least below $1 \mathrm{~Hz}$, i.e. so small that the displacement currents $(=\varepsilon \partial \mathrm{E} / \partial \mathrm{t})$ can practically always be neglected. It means that "geoelectromagnetic plane waves" are actually not "waves", so the terminology generally used in geoelectromagnetics is not completely correct in this respect.

Let us assume now that the Earth is uniform with the conductivity $\sigma$ and consider a harmonic time dependence with the angular frequency $\omega$. It is easy to show that the horizontal geoelectric field component $E_{y}=E_{y}(\omega)$ at the Earth's surface is related to the perpendicular horizontal geomagnetic variation component $B_{x}=B_{x}(\omega)$ by the following equation (e.g. Pirjola, 1982)

$$
E_{y}=-\sqrt{\frac{\omega}{\mu_{0} \sigma}} e^{i \frac{\pi}{4}} B_{x}
$$

Equation (10) shows that there is a 45-degree ( $\pi / 4$-radian) phase shift between the geoelectric and geomagnetic fields. We also see that an increase of the angular frequency and a decrease of the Earth's conductivity enhance the geoelectric field with respect to the geomagnetic field.

Noting that $\mathrm{i} \omega B_{x}(\omega)$ is associated with the time derivative of $B_{x}(t)$, equation (10) can be inverse-Fourier transformed to give the following time domain convolution integral (Cagniard, 1953; Pirjola, 1982)

$$
E_{y}(t)=-\frac{1}{\sqrt{\pi \mu_{0} \sigma}} \int_{0}^{\infty} \frac{g(t-u)}{\sqrt{u}} d u
$$

where the time derivative of $B_{x}(t)$ is denoted by $g(t)$. The derivation of equation (10) makes use of the neglect of the displacement currents, which thus also affects equation (11). If the displacement currents are included the kernel function convolved with $g(t)$ in formula (11) is more complicated containing the Bessel function of the zero order (Pirjola, 1982). Equation (11) is in agreement with the causality, i.e. $E_{y}(t)$ at the time $t$ only depends on earlier values of $g(t)$. The square root of the lag time $u$ in the denominator means that the influence of a value of $g(t-u)$ on $E_{y}(t)$ decreases with increasing $u$. As indicated in Section 2.1, equation (11) shows that the relation of the geoelectric field (and GIC) with the geomagnetic time derivative is not simple, like for example a proportionality, not even in the present planewave and uniform-Earth situation.

Similarly to the inverse-Fourier transform of equation (10) leading to (11) in the time domain, we can inverse-Fourier transform equation (9) to get a convolution relation between $E_{y}$ and $B_{x}$ in the time domain, or as above in equation (11), perhaps rather between $E_{y}$ and $\mathrm{d} B_{x} / \mathrm{d} t$.

The engineering part of GIC modelling utilises the horizontal geoelectric field to be provided by the geophysical part and also needs the knowledge of the topology, 
configuration and all resistance values of the network in question. The computation of GIC can be performed by applying electric circuit theory, i.e. Ohm's and Kirchhoff's laws. Because the frequencies in connection with geoelectromagnetic fields and GIC are very low, a dc treatment is appropriate to the engineering part (at least as the first approximation). Discretely-earthed networks, such as a power system, and continuously-earthed networks, such as a buried pipeline, need to have different calculation techniques. For the former, matrix formulas are available, which enable the computation of GIC between the Earth and the network at the nodes and in the lines between the nodes, whereas the latter can be treated by utilising the distributed-source transmission line (DSTL) theory. These methods are discussed more in Section 3.

Assuming that the horizontal geoelectric field impacting a network is uniform and applying the engineering part techniques, we can easily identify the sites that will most probably experience the largest GIC magnitudes being thus risky for problems, but being also ideal GIC recording sites. With a uniform geoelectric field, we may also simply get a comprehension of the effect of disconnecting or connecting some lines on GIC values in the network. Performing both the geophysical and the engineering parts enables studies of GIC as functions of time at different sites of the system during large space weather storms. Using long-term geomagnetic data recorded at observatories and other magnetometer stations, it is possible to derive statistics of expected GIC values at different sites of a technological network.

\section{Calculation of GIC}

\subsection{Power networks}

For investigating the engineering part of GIC modelling in the case of a power system, we consider a network of conductors with $N$ discrete nodes, called stations and earthed by the resistances $R_{e, i}(i=1, \ldots, N)$. Let us assume that the network is impacted by a horizontal geoelectric field E, which implies the flow of geomagnetically induced currents (GIC). Lehtinen and Pirjola (1985) derive a formula for the $N \times 1$ column matrix $\mathbf{I}_{\mathbf{e}}$ that includes the currents $I_{e, m}(m=1, \ldots, N)$ called earthing currents or earthing GIC and flowing between the network and the Earth as follows

$$
I_{e}=\left(1+Y_{n} Z_{e}\right)^{-1} J_{e}
$$

The current is defined to be positive when it flows from the network to the Earth and negative when it flows from the Earth to the network. The symbol 1 denotes the $N \times N$ unit identity matrix. The $N \times N$ earthing impedance matrix $\mathbf{Z}_{\mathbf{e}}$ and the $N \times N$ network admittance matrix $\mathbf{Y}_{\mathbf{n}}$ as well as the $N \times 1$ column matrix $\mathbf{J}_{\mathbf{e}}$ are explained below.

The definition of $\mathbf{Z}_{\mathbf{e}}$ states that multiplying the earthing current matrix $\mathbf{I}_{\mathbf{e}}$ by $\mathbf{Z}_{\mathbf{e}}$ gives the voltages between the earthing points and a remote Earth that are related to the flow of the currents $I_{e, m}(m=1, \ldots, N)$. Thus, expressing the voltages by an $N \times 1$ column matrix $\mathbf{U}$, we have

$$
\mathbf{U}=\mathbf{Z}_{\mathbf{e}} \mathbf{I}_{\mathbf{e}}
$$

Utilising the reciprocity theorem, $\mathbf{Z}_{\mathrm{e}}$ can be shown to be a symmetric matrix. The diagonal elements of $\mathbf{Z}_{\mathrm{e}}$ equal the earthing resistances of the stations. If the distances of the stations 
are large enough, the influence of the current $I_{e, m}$ at one station on the voltages at other stations is negligible, and then the off-diagonal elements of $\mathbf{Z}_{\mathbf{e}}$ are zero (see Pirjola, 2008).

The matrix $\mathbf{Y}_{\mathbf{n}}$ is defined by

$$
(i \neq m): Y_{n, i m}=-\frac{1}{R_{n, i m}},(i=m): Y_{n, i m}=\sum_{k=1, k \neq i}^{N} \frac{1}{R_{n, i k}}
$$

where $R_{n, i m}$ is the resistance of the conductor between stations $i$ and $m(i, m=1, \ldots, N)$. (If stations $i$ and $m$ are not directly connected by a conductor, $R_{n, i m}$ naturally gets an infinite value.) It is seen from equation (14) that $\mathbf{Y}_{\mathbf{n}}$ is symmetric.

The elements $J_{e, m}(m=1, \ldots, N)$ of the column matrix $\mathbf{J}_{\mathbf{e}}$ are given by

$$
J_{e, m}=\sum_{i=1, i \neq m}^{N} \frac{V_{i m}}{R_{n, i m}}
$$

The geovoltage $V_{i m}$ is produced by the horizontal geoelectric field $\mathbf{E}$ along the path defined by the conductor line from station $i$ to station $m(i, m=1, \ldots, N)$, i.e.

$$
V_{m}=\int_{i}^{m} \mathbf{E} \cdot d \mathbf{s}
$$

Generally, the horizontal geoelectric field is rotational, i.e. the vertical component of the curl of $\mathbf{E}$ is not zero. As seen from equation (3), this is the case when the time derivative of the vertical component of the magnetic field differs from zero. Consequently, the integral in equation (16) is path-dependent. Thus, as indicated, the integration route must follow the conductor between $i$ and $m$ (Boteler and Pirjola, 1998b; Pirjola, 2000). Equations (15) and (16) show that the column matrix $\mathbf{J}_{\mathbf{e}}$ involves the contribution from the geoelectric field $\mathbf{E}$ to equation (12). Note that $\mathbf{I}_{\mathbf{e}}$ and $\mathbf{J}_{\mathbf{e}}$ are equal in the case of perfect earthings, i.e. when $\mathbf{Z}_{\mathbf{e}}=0$.

Pirjola (2007) presents an alternative, but equivalent, expression for the matrix $\mathbf{I}_{\mathbf{e}}$, which includes the geovoltages more explicitly. It makes use of the total admittance matrix that is defined to be the sum of $\mathbf{Y}_{\mathrm{n}}$ and the inverse of $\mathbf{Z}_{\mathrm{e}}$. However, the application of this alternative expression obviously does not produce any noticeable advantages, for example, in numerical computations.

So far, we have discussed GIC flowing between the network and the Earth. There is also a simple expression for GIC in the conductors between the nodes (e.g. Pirjola, 2007). However, we ignore it and concentrate only on earthing GIC because, in the case of a power system, GIC to (or from) the Earth constitute the harmful currents in transformer windings and they are usually measured (see Section 2.1).

When GIC in a power network are calculated, the three phases are usually treated as one circuit element. The resistance of the element is then one third of that of a single phase, and the GIC flowing in the element is three times the current in a single phase. Moreover, the earthing resistances (which might be called the total earthing resistances) are convenient to be assumed to include the actual earthing resistances, the transformer resistances and the 
resistances of possible reactors or any resistors in the earthing leads of transformer neutrals, with all these resistances connected in series.

As Mäkinen (1993) and Pirjola (2005) present, special modelling techniques are needed for transformers when two different voltage levels are considered in the calculation of GIC in a power network. Moreover, the treatment of autotransformers differs from the case of full two-winding transformers. It is worth mentioning that recent, still unpublished, investigations indicate that the methods and equations described by Mäkinen (1993) and Pirjola (2005) are unnecessarily complicated, though correct.

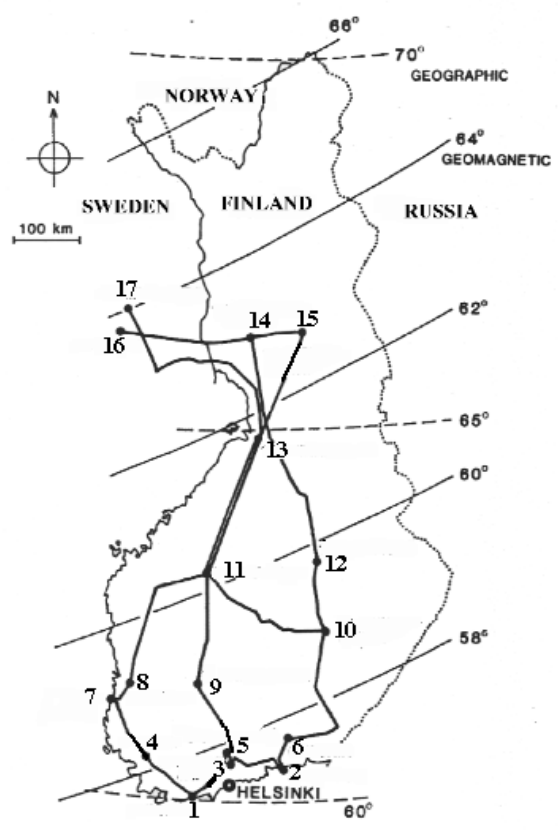

Fig. 3. Finnish 400-kV electric power transmission grid in its configuration valid in October 1978 to November 1979 (Pirjola \& Lehtinen, 1985; Pirjola, 2005; Pirjola, 2009). This network can be used as a "test model" for GIC calculation algorithms and programs.

Pirjola (2009) introduces an old version of Finland's $400 \mathrm{kV}$ power network as a "test model" for GIC computation algorithms and programs. The network is shown in Figure 3. It consists of 17 stations and 19 transmission lines, so that it is complex enough to reveal essential features included in GIC computations but not too large to unnecessarily complicate the calculations and analyses. Pirjola (2009) provides the (Cartesian) north and east coordinates of the locations of the stations included in the test model and numbered from 1 to 17. The (total) earthing resistances of the stations as well as the resistances of the transmission lines are also given. Regarding the use of the network as a test model, Pirjola (2009) presents GIC values at all stations and in every transmission line that a uniform (geographically) eastward or a uniform (geographically) northward geoelectric field of 1 
$\mathrm{V} / \mathrm{km}$ creates. Here it should be noted that, by using a linear superposition, these data enable the computation of GIC due to any uniform horizontal geoelectric field impacting the network. For some additional details about the test model, Pirjola (2009) is referred to.

The Rauma $400 \mathrm{kV}$ station discussed in Section 2.1 and especially in Figure 1 was not yet included in the Finnish $400 \mathrm{kV}$ system in October 1978 to November 1979. It is located in the line between stations 4 and 7 quite near station 7 . Note that geographic and geomagnetic latitudes are far from being parallel in Finland and that the former are higher. In North America, the geographic latitudes are lower than the geomagnetic since the north geomagnetic pole is on the American side of the geographic pole.

\subsection{Pipelines}

Pirjola \& Lehtinen (1985) present theoretical computations of GIC for the Finnish natural gas pipeline by using the matrix formalism discussed in Section 3.1 and appropriate to a discretely-earthed network, such as a power system. The assumption is included in the treatment by Pirjola \& Lehtinen (1985) that the insulating coating of the pipeline is ideal and perfect with a zero conductivity and that the pipeline is earthed at the cathodic protection stations. This approximation should not be considered very good because the large surface area of the pipe makes the pipeline continuously earthed in practice even though the conductivity of the coating material is very small. Furthermore, the CP stations do not constitute normal earthings since the current can only go to the Earth there (to return from the ground to the pipe elsewhere).

Viljanen (1989) presents a GIC study about the Finnish natural gas pipeline based on the simplified assumption that the pipeline is an infinitely long multi-layered cylindrical structure in a homogeneous medium. The model is in agreement with the continuous earthing but it is otherwise too much idealised. According to this model, GIC flowing along the pipeline may reach values of hundreds of amps, which are clearly larger than those measured in Finland (Section 2.1). Although Viljanen (1989) also estimates the effects of a horizontal change of the Earth's conductivity, and of a bend of the pipeline, the treatment is not yet complete for a real pipeline network. A significant improvement in theoretical modelling is shown by Boteler (1997) by incorporating the distributed-source transmission line theory into pipeline-GIC calculations. In fact, the applicability of the DSTL theory is already considered by Boteler and Cookson (1986). An extension is provided by Pulkkinen et al. (2001b) as they also treat branches of a pipeline network.

In the DSTL theory, the pipeline is considered a transmission line containing a series impedance (or resistance due to the dc treatment) $Z$ determined by the properties of the pipeline steel and a parallel admittance $Y$ associated with the resistivity of the coating. The geoelectric field affecting the pipeline forms the distributed source. An important parameter, called the adjustment distance, is the inverse of the propagation constant $\gamma$ defined by

$$
\gamma=\sqrt{Z Y}
$$

Typical values of the adjustment distance of a real pipeline are tens of kilometres. For the Finnish natural gas pipeline, $Z=5 \ldots 9 \cdot 10^{-3} \Omega \mathrm{km}^{-1}$ and $Y=5 \cdot 10^{-2} \ldots 0.25 \Omega^{-1} \mathrm{~km}^{-1}$ with the 
exact values depending on the radius of the pipeline at the particular section. Thus, the adjustment distance has values from about $20 \mathrm{~km}$ to about $60 \mathrm{~km}$.

Concerning GIC and pipe-to-soil voltages, electrically long pipelines (length $>>$ adjustment distance) behave differently than electrically short pipes (length $<<$ adjustment distance). For a long pipeline, the voltage decays exponentially at a distance comparable to the adjustment distance, when moving from either end of the pipeline towards the centre where it is practically zero. In the same areas near the ends, GIC flows between the pipe and the soil, and in the central parts the current along the pipe is spatially constant. For a short pipeline, the voltage changes linearly along the pipeline, and the current along the pipe is small when the ends are insulated from the Earth. Consequently, central parts of a long pipeline are not critical regarding corrosion problems due to GIC. On the other hand, the pipe-to-soil voltages remain smaller in short pipelines than the end voltages of a long pipeline. Therefore, it might sometimes be reasonable to install insulating flanges in series in a pipeline to break it into shorter sections.

The handling of inhomogeneities associated with a pipeline, such as bends, changes of the pipeline material or of the pipeline size, and branches of the pipeline network, can be accomplished by applying Thévenin's theorem. It expresses an equivalent voltage source and an impedance that describe an external circuit at the terminals of a network section considered. Thus at a pipeline inhomogeneity, Thévenin's voltage and impedance of a section terminating at the inhomogeneity have to be calculated when considering GIC and pipe-to-soil voltages in another section. In this way it is possible to go through a whole pipeline network from section to section. Branches require a special treatment since, for a particular section, Thévenin's components of the other sections are connected in parallel (Pulkkinen et al., 2001b).

\section{Conclusion}

Geomagnetically induced currents (GIC) are ground effects of space weather, which is associated with a complex chain of phenomena extending from processes in the Sun to GIC in technological networks. In general, GIC are a possible source of problems to the network. Thus research of GIC is practically important, but it also has scientific significance because a ground-based network carrying GIC can be regarded as a huge antenna that collects information from processes in space and within the Earth.

The first GIC observations date back to early telegraph systems more than 150 years ago. Today power systems constitute the most important target of GIC research. The problems in power networks result from half-cycle saturation of transformers created by dc-like GIC. In the worst cases, large areas may experience a blackout due to GIC and transformers can be permanently damaged. The most significant GIC event so far is the blackout in Québec, Canada, for several hours during a large geomagnetic storm in March 1989. Another well-known GIC event caused a blackout in southern Sweden during the so-called "Halloween storm" at the end of October 2003. In 2011 to 2014, an EU-funded project is going on in which GIC in the entire European high-voltage system are considered.

In this paper, we discuss the techniques readily available for calculating GIC values in power networks and pipelines. Future research efforts should be focussed on the application 
of the methods to data characterising extremely large space weather events, such as the famous Carrington storm in 1859. Such studies would lead to extreme, but realistic, scenarios of GIC magnitudes to be utilised in the estimation of possible problems and in the design of countermeasures.

\section{Acknowledgment}

The author wishes to thank his colleagues for excellent collaboration in GIC research during many years. Special thanks go to Drs. David Boteler (Canada), Antti Pulkkinen (Finland \& USA), Larisa Trichtchenko (Canada), Ari Viljanen (Finland) and Magnus Wik (Sweden) (in alphabetical order). The author also wants to acknowledge the interest and support that Finnish power and pipeline industry have shown to GIC investigations during more than thirty years.

\section{References}

Bernhardi, E. H.; Cilliers, P. J. \& Gaunt, C. T. (2008). Improvement in the modelling of geomagnetically induced currents in southern Africa. South African Journal of Science, Vol.104, July/August 2008, pp. 265-272

Bolduc, L. (2002). GIC observations and studies in the Hydro-Québec power system. Journal of Atmospheric and Solar-Terrestrial Physics, Vol.64, No.16, pp. 1793-1802

Boteler, D. H. (1997). Distributed-Source Transmission Line Theory for Electromagnetic Induction Studies. Supplement of the Proceedings of the 12th International Zurich Symposium and Technical Exhibition on Electromagnetic Compatibility, Zürich, Switzerland, February 18-20, 1997, OE7, pp. 401-408

Boteler, D. \& Cookson, M. J. (1986). Telluric currents and their effects on pipelines in the Cook Strait region of New Zealand. Materials Performance, March 1986, pp. 2732

Boteler, D. H. \& Pirjola, R. J. (1998a). The complex-image method for calculating the magnetic and electric fields produced at the surface of the Earth by the auroral electrojet. Geophysical Journal International, Vol.132, No.1, pp. 31-40

Boteler, D. H. \& Pirjola, R. J. (1998b). Modelling Geomagnetically Induced Currents produced by Realistic and Uniform Electric Fields. IEEE Transactions on Power Delivery, Vol.13, No.4, pp. 1303-1308

Boteler, D. H.; Pirjola, R. J. \& Nevanlinna, H. (1998). The effects of geomagnetic disturbances on electrical systems at the earth's surface. Advances in Space Research, Vol.22, No.1, pp. 17-27

Cagniard, L. (1953). Basic theory of the magnetotelluric method of geophysical prospecting. Geophysics, Vol.18, pp. 605-635

Elovaara, J. (2007). Finnish experiences with grid effects of GIC's. In: Space Weather, Research towards Applications in Europe, J. Lilensten (Ed.), Astrophysics and Space Science Library, 344, ESA, COST 724, Springer, Chapter 5.4, pp. 311-326

Elovaara, J.; Lindblad, P.; Viljanen, A.; Mäkinen, T.; Pirjola, R.; Larsson, S. \& Kielén, B. (1992). Geomagnetically induced currents in the Nordic power system and their effects on equipment, control, protection and operation. CIGRÉ Paper No. 36-301 
(CIGRÉ = International Conference on Large High Voltage Electric Systems), CIGRÉ General Session 1992, Paris, France, August 31 - September 5, 1992, No. 36301, $10 \mathrm{pp}$.

Eroshenko, E. A.; Belov, A. V.; Boteler, D.; Gaidash, S. P.; Lobkov, S. L.; Pirjola, R. \& Trichtchenko, L. (2010). Effects of strong geomagnetic storms on Northern railways in Russia. Advances in Space Research, Vol.46, No.9, pp. 1102-1110

Gummow, R. A. (2002). GIC effects on pipeline corrosion and corrosion control systems. Journal of Atmospheric and Solar-Terrestrial Physics, Vol.64, No.16, pp. 17551764

Häkkinen, L. \& Pirjola, R. (1986). Calculation of electric and magnetic fields due to an electrojet current system above a layered earth. Geophysica, Vol.22, Nos.1-2, pp. 3144

Kappenman, J. G. (2003). Storm sudden commencement events and the associated geomagnetically induced current risks to ground-based systems at low-latitude and midlatitude locations. Space Weather, Vol.1, No.3, 1016, doi:10.1029/2003SW000009, $16 \mathrm{pp}$.

Kappenman, J. G. (2007). Geomagnetic Disturbances and Impacts upon Power System Operation. In: The Electric Power Engineering Handbook, L. L. Grigsby (Ed.), CRC Press/IEEE Press, 2nd Edition, Chapter 16, pp. 16-1 - 16-22

Kappenman, J. G. \& Albertson, V. D. (1990). Bracing for the geomagnetic storms. IEEE Spectrum, March 1990, pp. 27-33

Kaufman, A. A. \& G. V. Keller, G. V. (1981). The Magnetotelluric Sounding Method, Methods in Geochemistry and Geophysics, 15, Elsevier Scientific Publishing Company, 595 pp.

Lanzerotti, L. J. (2010). Using the Guide of History, Space Weather, Vol.8, S03004, doi:10.1029/2010SW000579, 2 pp.

Lanzerotti, L. J.; Thomson, D. J. \& Maclennan, C. G. (1999). Engineering issues in space weather, In: Modern Radio Science 1999, M. A. Stuchly (Ed.), International Union of Radio Science (URSI), Oxford University Press, pp. 25-50

Lehtinen, M. \& Pirjola, R. (1985). Currents produced in earthed conductor networks by geomagnetically-induced electric fields. Annales Geophysicae, Vol.3, No.4, pp. 479484

Liu, C.-M.; Liu, L.-G. \& Pirjola, R. (2009a). Geomagnetically Induced Currents in the HighVoltage Power Grid in China. IEEE Transactions on Power Delivery, Vol.24, No.4, pp. 2368-2374

Liu, C.-M.; Liu, L.-G.; Pirjola, R. \& Wang, Z.-Z. (2009b). Calculation of geomagnetically induced currents in mid- to low-latitude power grids based on the plane wave method: A preliminary case study. Space Weather, Vol.7, No.4, S04005, doi: 10.1029/2008SW000439, 9 pp.

Mäkinen, T. (1993). Geomagnetically induced currents in the Finnish power transmission system. Finnish Meteorological Institute, Geophysical Publications, No.32, Helsinki, Finland, $101 \mathrm{pp}$.

Molinski, T. S. (2002). Why utilities respect geomagnetically induced currents. Journal of Atmospheric and Solar-Terrestrial Physics, Vol.64, No.16, pp. 1765-1778 
Pirjola, R. (1982). Electromagnetic induction in the earth by a plane wave or by fields of line currents harmonic in time and space. Geophysica, Vol.18, Nos.1-2, pp. $1-161$

Pirjola, R. (2000). Geomagnetically Induced Currents During Magnetic Storms. IEEE Transactions on Plasma Science, Vol.28, No.6, pp. 1867-1873

Pirjola, R. (2002). Review on the calculation of surface electric and magnetic fields and of geomagnetically induced currents in ground-based technological systems. Surveys in Geophysics, Vol.23, No.1, pp. 71-90

Pirjola, R. (2005). Effects of space weather on high-latitude ground systems. Advances in Space Research, Vol.36, No.12, doi: 10.1016/j.asr.2003.04.074, pp. 2231-2240

Pirjola, R. (2007). Calculation of geomagnetically induced currents (GIC) in a high-voltage electric power transmission system and estimation of effects of overhead shield wires on GIC modelling. Journal of Atmospheric and Solar-Terrestrial Physics, Vol.69, No.12, pp. 1305-1311

Pirjola, R. (2008). Effects of interactions between stations on the calculation of geomagnetically induced currents in an electric power transmission system. Earth, Planets and Space, Vol.60, No.7, pp. 743-751

Pirjola, R. (2009). Properties of matrices included in the calculation of geomagnetically induced currents (GICs) in power systems and introduction of a test model for GIC computation algorithms. Earth, Planets and Space, Vol.61, No.2, pp. 263272

Pirjola, R. (2010). Derivation of characteristics of the relation between geomagnetic and geoelectric variation fields from the surface impedance for a two-layer earth. Earth, Planes and Space, Vol.62, No.3, pp. 287-295

Pirjola, R. J. \& Boteler, D. H. (2006). Geomagnetically induced currents in European highvoltage power systems. CD ROM Proceedings of the Canadian Conference on Electrical and Computer Engineering (CCECE), IEEE Ottawa, Ottawa, Canada, May 7-10, 2006, Paper 820, 4 pp.

Pirjola, R. J. \& Häkkinen, L. V. T. (1991). Electromagnetic Field Caused by an Auroral Electrojet Current System Model. In: Environmental and Space Electromagnetics, H. Kikuchi (Ed.), Springer-Verlag, Tokyo, Printed in Hong Kong, Chapter 6.5, pp. 288298

Pirjola, R. \& Lehtinen, M. (1985). Currents produced in the Finnish $400 \mathrm{kV}$ power transmission grid and in the Finnish natural gas pipeline by geomagneticallyinduced electric fields. Annales Geophysicae, Vol.3, No.4, pp. 485-491

Pirjola, R. \& Viljanen, A. (1998). Complex image method for calculating electric and magnetic fields produced by an auroral electrojet of finite length. Annales Geophysicae, Vol.16, No.11, pp. 1434-1444

Pirjola, R.; Boteler, D. \& Trichtchenko, L. (2009). Ground effects of space weather investigated by the surface impedance. Earth, Planets and Space, Vol.61, No.2, pp. 249-261.

Pirjola, R.; Pulkkinen, A. \& Viljanen, A. (2003), Studies of space weather effects on the Finnish natural gas pipeline and on the Finnish high-voltage power system, Advances in Space Research, Vol.31, No.4, pp. 795-805 
Pirjola, R.; Kauristie, K.; Lappalainen, H.; Viljanen, A. \& Pulkkinen, A. (2005). Space weather risk. Space Weather, Vol.3, No.2, S02A02, doi: 10.1029/2004SW000112, 11 pp.

Ptitsyna, N. G.; Kasinskii, V. V.; Villoresi, G.; Lyahov, N. N.; Dorman, L. I. \& Iucci, N. (2008). Geomagnetic effects on mid-latitude railways: A statistical study of anomalies in the operation of signaling and train control equipment on the East-Siberian Railway. Advances in Space Research, Vol.42, No.9, pp. 1510-1514

Pulkkinen, A.; Viljanen, A.; Pajunpää, K. \& Pirjola, R. (2001a). Recordings and occurrence of geomagnetically induced currents in the Finnish natural gas pipeline network. Journal of Applied Geophysics, Vol. 48, No.4, pp. 219-231

Pulkkinen, A.; Pirjola, R.; Boteler, D.; Viljanen, A. \& Yegorov, I. (2001b). Modelling of space weather effects on pipelines. Journal of Applied Geophysics, Vol.48, No.4, pp. 233256

Trichtchenko, L. \& Boteler, D. H. (2006). Response of power systems to the temporal characteristics of geomagnetic storms. CD ROM Proceedings of the Canadian Conference on Electrical and Computer Engineering (CCECE), IEEE Ottawa, Ottawa, Canada, May 7-10, 2006, Paper 387, 4 pp.

Trichtchenko, L. \& Boteler, D. H. (2007). Effects of recent geomagnetic storms on power systems, Proceedings of the 7-th International Symposium on Electromagnetic Compatibility and Electromagnetic Ecology, Saint-Petersburg, Russia, June 26-29, 2007, pp. 265-268

Trivedi, N. B.; Vitorello, Í.; Kabata, W.; Dutra, S. L. G.; Padilha, A. L.; Bologna, M. S.; de Pádua, M. B.; Soares, A. P.; Luz, G. S.; de A. Pinto, F.; Pirjola, R. \& Viljanen, A. (2007). Geomagnetically induced currents in an electric power transmission system at low latitudes in Brazil: A case study. Space Weather, Vol.5, No.4, S04004, doi: 10.1029/2006SW000282, 10 pp.

Viljanen, A. (1989). Geomagnetically Induced Currents in the Finnish Natural Gas Pipeline. Geophysica, Vol.25, Nos.1\&2, pp. 135-159

Viljanen, A. T., Pirjola, R. J.; Pajunpää, K. M. \& Pulkkinen, A. A. (2009). Measurements of geomagnetically induced currents by using two magnetometers. Proceedings of the 8-th International Symposium on Electromagnetic Compatibility and Electromagnetic Ecology, Saint-Petersburg, Russia, June 16-19, 2009, pp. 227-230

Viljanen, A.; Pulkkinen, A.; Amm, O.; Pirjola, R.; Korja, T. \& BEAR Working Group (2004). Fast computation of the geoelectric field using the method of elementary current systems and planar Earth models. Annales Geophysicae, Vol.22, No.1, pp. 101113

Viljanen, A.; Pulkkinen, A.; Pirjola, R.; Pajunpää, K.; Posio, P. \& Koistinen, A. (2006). Recordings of geomagnetically induced currents and a nowcasting service of the Finnish natural gas pipeline system. Space Weather, Vol.4, No.10, S10004, doi: 10.1029/2006SW000234, 9 pp.

Watari, S.; Kunitake, M.; Kitamura, K.; Hori, T.; Kikuchi, T.; Shiokawa, K.; Nishitani, N.; Kataoka, R.; Kamide, Y.; Aso, T.; Watanabe, Y. \& Tsuneta, Y. (2009). Measurements of geomagnetically induced current in a power grid in Hokkaido, Japan. Space Weather, Vol.7, No.3, S03002, doi: 10.1029/2008SW000417, 11 pp.

Wik, M.; Pirjola, R.; Lundstedt, H.; Viljanen, A.; Wintoft, P. \& Pulkkinen, A. (2009). Space weather events in July 1982 and October 2003 and the effects of geomagnetically 
induced currents on Swedish technical systems. Annales Geophysicae, Vol.27, No.4, pp. $1775-1787$

Wik, M.; Viljanen, A.; Pirjola, R.; Pulkkinen, A.; Wintoft, P. \& Lundstedt, H. (2008). Calculation of geomagnetically induced currents in the $400 \mathrm{kV}$ power grid in southern Sweden. Space Weather, Vol.6, No.7, S07005, doi: 10.1029/2007SW000343, $11 \mathrm{pp}$. 


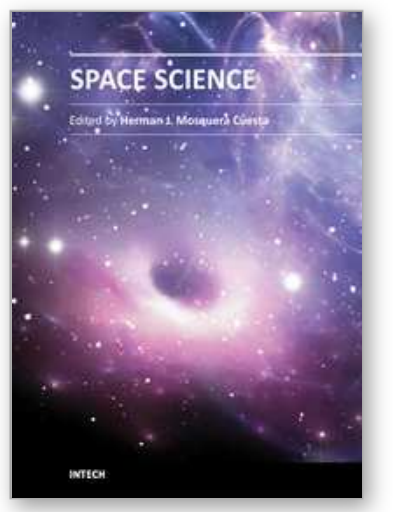

\author{
Space Science \\ Edited by Dr. Herman J. Mosquera Cuesta
}

ISBN 978-953-51-0423-0

Hard cover, 152 pages

Publisher InTech

Published online 23, March, 2012

Published in print edition March, 2012

The all-encompassing term Space Science was coined to describe all of the various fields of research in science: Physics and astronomy, aerospace engineering and spacecraft technologies, advanced computing and radio communication systems, that are concerned with the study of the Universe, and generally means either excluding the Earth or outside of the Earth's atmosphere. This special volume on Space Science was built throughout a scientifically rigorous selection process of each contributed chapter. Its structure drives the reader into a fascinating journey starting from the surface of our planet to reach a boundary where something lurks at the edge of the observable, light-emitting Universe, presenting four Sections running over a timely review on space exploration and the role being played by newcomer nations, an overview on Earth's early evolution during its long ancient ice age, a reanalysis of some aspects of satellites and planetary dynamics, to end up with intriguing discussions on recent advances in physics of cosmic microwave background radiation and cosmology.

\title{
How to reference
}

In order to correctly reference this scholarly work, feel free to copy and paste the following:

Risto Pirjola (2012). Geomagnetically Induced Currents as Ground Effects of Space Weather, Space Science, Dr. Herman J. Mosquera Cuesta (Ed.), ISBN: 978-953-51-0423-0, InTech, Available from:

http://www.intechopen.com/books/space-science/geomagnetically-induced-currents-as-ground-effects-ofspace-weather

\section{INTECH}

open science | open minds

\section{InTech Europe}

University Campus STeP Ri

Slavka Krautzeka 83/A

51000 Rijeka, Croatia

Phone: +385 (51) 770447

Fax: +385 (51) 686166

www.intechopen.com

\section{InTech China}

Unit 405, Office Block, Hotel Equatorial Shanghai

No.65, Yan An Road (West), Shanghai, 200040, China

中国上海市延安西路65号上海国际贵都大饭店办公楼405单元

Phone: +86-21-62489820

Fax: +86-21-62489821 
(C) 2012 The Author(s). Licensee IntechOpen. This is an open access article distributed under the terms of the Creative Commons Attribution 3.0 License, which permits unrestricted use, distribution, and reproduction in any medium, provided the original work is properly cited. 